\title{
Free-Shaped Object Recognition Method from Partial Views Using Weighted Cone Curvatures
}

\author{
Santiago Salamanca ${ }^{1}$, Carlos Cerrada ${ }^{2}$, Antonio Adán ${ }^{3}$, \\ Jose A. Cerrada ${ }^{2}$, and Miguel Adán ${ }^{4}$ \\ ${ }^{1}$ Escuela de Ingenierías Industriales, Universidad de Extremadura, \\ Av. Elvas s/n, 06071 Badajoz, Spain \\ ssalaman@unex.es \\ ${ }^{2}$ Escuela Técnica Superior de Ingeniería Informática, \\ UNED juan del rosal 16, 28040 Madrid, Spain \\ \{ccerrada, jcerrada\}@issi.uned.es \\ ${ }^{3}$ Escuela Superior de Informática, Universidad de Castilla La Mancha, \\ Paseo de la Universidad 4, 13071 Ciudad Real, Spain \\ antonio.adan@uclm.es \\ ${ }^{4}$ Escuela de Ingeniería Técnica Agrícola, Universidad de Castilla la Mancha, \\ Ronda de Calatrava n $^{\circ}$ 7, 13071 Ciudad Real, Spain \\ miguel.adan@uclm.es
}

\begin{abstract}
This work presents a method for free-shaped object recognition from its partial views. Consecutive database reductions are achieved in three stages by using effective discriminant features. These features are extracted from the spherical mesh representation used to modeling the partial view and from the view range data itself. The used characteristics are global, which means that they can not represent the views univocally. However, their staged application allows the initial object database to be reduced to selecting just one candidate in the final stage with a high success rate. Yet, the most powerful search reduction is achieved in the first stage where the new Weighted Cone Curvature (WCC) parameter is processed. The work is devoted to describe the overall method making especial emphasis in the WCC feature and its application to partial views recognition. Results with real objects range data are also presented in the paper.
\end{abstract}

\section{Introduction}

The recognition problem tries to identify an object, called unknown or scene object, from a set of objects in an object database, generally called models. The problem of positioning or alignment solves the localization of an object in a scene with respect to a reference system linked to the model of this object. One of the most common ways of solving this problem is matching the unknown object on the corresponding object in the object database.

Although conceptually recognition and positioning are two different problems, in practice they are closely related. If we can align the unknown object precisely on one of the different objects in the database, we will have solved not only positioning but also recognition. 
The case that we present in this work is recognition of the range data of a partial view by matching the view on the range data of the complete objects stored in the object database. The resolution of the problem is of real practical interest since it can be used in tasks with industrial robots, mobile robot navigation, visual inspection, etc.

In order to tackle the problem posed it is necessary to generate a model that allows us to extract information from the source data and represent them. Regarding these representations there are two fundamental categories: object-based representation and view-centered representation.

The first creates models based on representative characteristics of the objects $[8,5$, 3 ], while the second tries to generate the model according to the appearance of the object from different points of view [4, 7].

Some other methods $[6,9]$ are halfway between these two categories since they do not capture the appearance of the object from each point of view, but provide just a characteristic measurement of the object. This is our case since our basis will be different measurements on the meshes or on the range data of the objects, calculated from different points of view. In this particular direction is addressed the problem in [1] where a shape similarity measure is introduced and applied. Nevertheless, this solution does not solve satisfactorily the object recognition problem from real partial views, which is the main purpose of our method.

The structure of the work will be as follows: in section 2 we will do a general description of the three stages of method proposed. In section 3 we will study first stage and the WCC feature which is the key of our method. Some explanations of the two others stages are stated in section 4. Then, in section 5 we will give the experimental results of the method, making special emphasis in the high reduction rates achieved in the first stage, and in section 6 the conclusions of this work.

\section{Overall Method: Functioning Principle}

The method presented in this work obtains effective database reduction by applying sequentially different global characteristics calculated on the spherical meshes and the range data of partial views (Fig. 1).

In the first stage we use a new invariant that we call Weihgted Cone-Curvature $(W C C)$ to determine a first approximation to the possible axes of vision from which this partial view has been acquired. Discretization of the vision space is obtained by circumscribing a spherical mesh around the model of the complete object. Each node in this mesh, together with the origin of coordinates, defines the initial axes of vision around this model. Therefore, determining the possible axes of vision from which the partial view has been acquired is equivalent to selecting a set of nodes on the mesh and rejecting the others. It is important to bear in mind that with this reduction what we are doing implicitly is a reduction of the possible rotations that could be applied on the partial view to match it on the model of the complete object.

We will call the nodes obtained after this first step $\mathbf{N}_{i}^{c c} \subset \mathbf{N}_{i}$, where $\mathbf{N}_{i}$ are the initial nodes of the spherical mesh circumscribed in the $i$-th object of the database. As is deduced from the explanation, in this stage the number of models in the object database is not reduced. 
Another invariant based on the principal components (eigen values + eigen vectors) of the partial view and complete object range data will be applied in a second stage on the selected nodes. After this features comparison a list for each of the objects in the database will be created with the nodes $\mathbf{N}_{i}^{c c}$ ordered according to the error existing in the eigen values comparison. This ordering in turn means that it is possible to identify which object has the greatest probability of matching the partial view. At the end of this second stage a reduction of the models in the object database is obtained together with the reduction of the nodes determined in the previous stage. If we call the initial object database $\mathbf{B}$, the base obtained after comparing the eigen values will be $\mathbf{B}^{c p} \subset \mathbf{B}$, and the nodes for each object $\mathbf{N}_{i}^{c p} \subset \mathbf{N}_{i}^{c c}$.

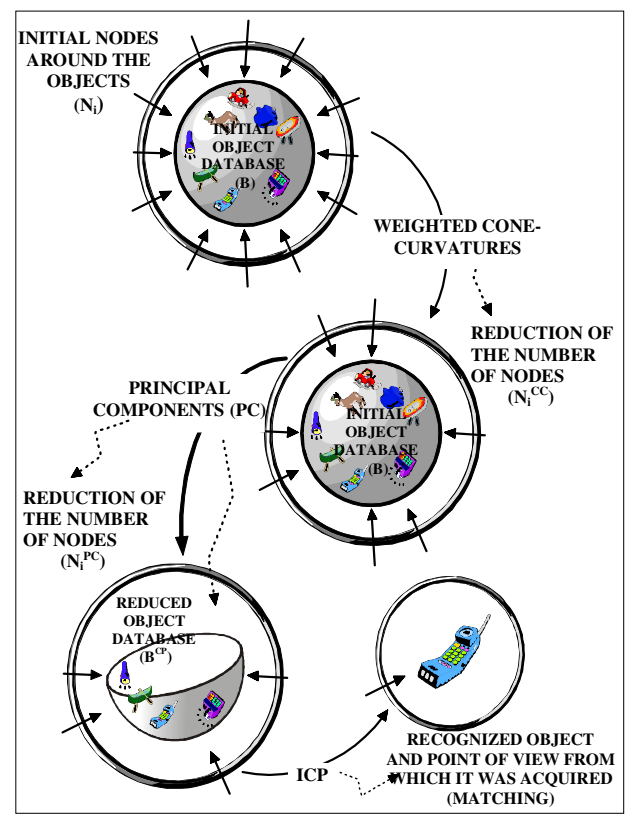

Fig. 1. Scheme of the different stages of the method for object recognition from partial views:graphical representation

The eigen vectors will allow a first approximation to be done to the rotation existing between the partial view and each one of the objects of $\mathbf{B}^{c p}$, which will be used in the last stage when the Iterative Closest Point (ICP) algorithm is applied. This allows the matching to be done between the range data, and the convergence error of the algorithm will indicate the object to which the partial view belongs.

The last two stages of the method are based on conventional techniques, whereas the first one represents a novel way of dealing with this problem. Therefore, only this key stage of the applied method will be detailed in next section. 


\section{First Stage: Robust Determination of the Point of View}

As it has been mentioned, in this stage of the recognition method the possible points of view from which the partial view has been acquired are estimated. For this task a new characteristic is proposed. Some preliminary concepts must be introduced previously to present this feature. It is calculated from partial spherical model, which we will call $T^{\prime}$, created from the range data of a given partial view. The partial spherical modeling technique is not covered in this work. After, a mesh adjusted to the range data is obtained whose patches are hexagonal or pentagonal. Each of the nodes of the mesh has a connectivity of 3 except for those nodes that are in the contour with connectivity less than 3 . Fig, 2 shows the intensity image of an object and the spherical model for a partial view of the object.
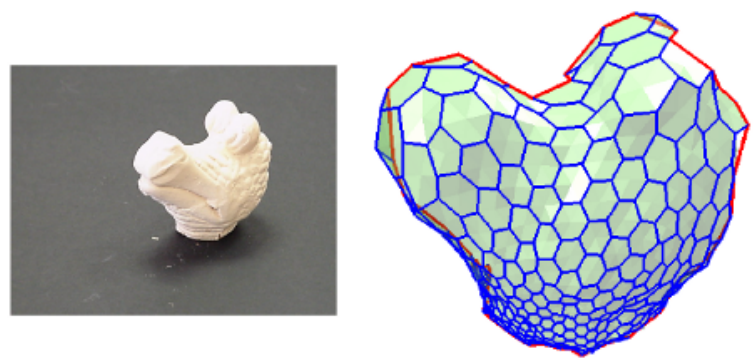

Fig. 2. Intensity image of an object and spherical model for a partial view of the object

A structure called Modeling Wave Set (MWS) [2] can be created on the spherical model, which is generated from a simpler structure called Modeling Wave (MW). Any node of the complete spherical mesh $T$ can be a focus and a MW can therefore be generated from it. Thus, a tessellated sphere with a number of nodes $n=\operatorname{ord}(T)$ will contain $n$ MW's. Fig. 3 shows two MW's on the tessellated sphere and the axes of vision defined by the focus and origin of coordinates. Notice that each MW is composed of several Wave Fronts $\left(F^{j}\right)$.
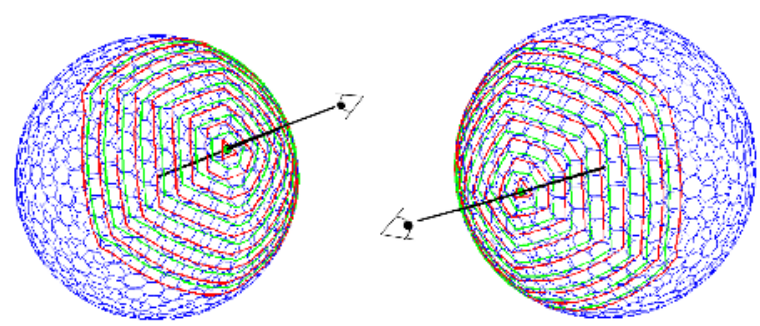

Fig. 3. Representation of two different modeling wave on the tessellated sphere

In this point the concept of Cone-Curvature (CC) is used. The CC is a characteristic calculated for each node of the mesh from the MWS. Its definition and 
main features are stated in [1]. For each wave front $F^{j}$ it determines an angle $\alpha^{j}$ and its range of values is $[-\pi / 2, \pi / 2]$. Negative values indicate the existence of concave areas; values near to zero correspond to flat surfaces, and positive values to convex areas. Fig. 4 illustrates the definition of Cone Curvature.

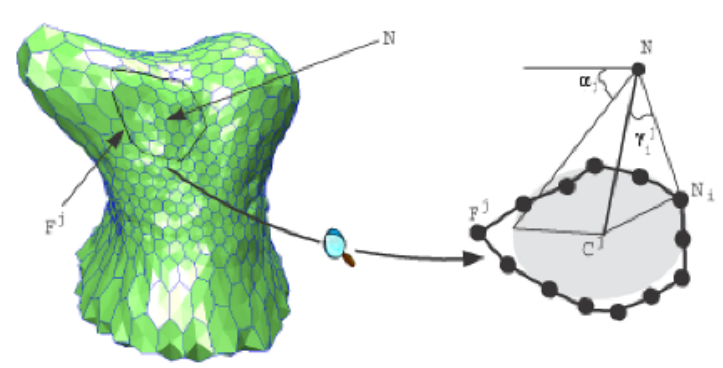

Fig. 4. Definition of the $\mathrm{j}$-th Cone Curvature (CC) for a given focus $N$

Given a focus $N$, there exists a set of $q$ wave fronts which define the CC's for this focus $\left\{\alpha^{l}, \ldots, \alpha^{q}\right\}$ and which provide complete information about the curvature of the existing object from the point of view that determines $N$. Finally, $q$, which is known as front order, can have values from 2 (case $q=1$ does not make sense) to the maximum number of fronts that the object has. As we want to work with partial models, the maximum order will be calculated for a number of wave fronts equal to the number of complete fronts in our partial model.

Main properties of CC's are their uniqueness (sufficient distant values for different objects), invariance to affine transformations- translation, rotation and scaling- (same values map for the same object shape) and robustness (higher orders CC's converge whereas important noise rates appearing in the model generation process). A deeper analysis of these properties can be found in [1], and it can be deduced that they are especially adequate to be applied to recognition tasks.

\subsection{Weighted Cone-Curvature (WCC)}

In this work we propose to use more compact information from the CC's with the purpose of reducing their dimensionality. This can be done by replacing each vector $\left\{\alpha^{l}, \ldots, \alpha^{q}\right\}$ calculated from $N^{\prime} \in T$ with a scalar linear combination of their components, denoted as $c^{w}$, that will characterize the object from this node. We will call this new global characteristic Weighted Cone-Curvature (WCC) and it is the key parameter to implement the reduction in the number of nodes.

To evaluate quantitatively if the reduction proposed is possible, the correlation existing in the CC's was calculated. This is shown in gray scale in Fig. 5 (white for high values and black for low values), and it has been calculated as the mean value of all the correlation coefficients of the meshes in the database. From analysis of the figure, and as we had envisaged, it can be concluded that there is a high correlation between fronts of near orders, which increases as this order increases. 


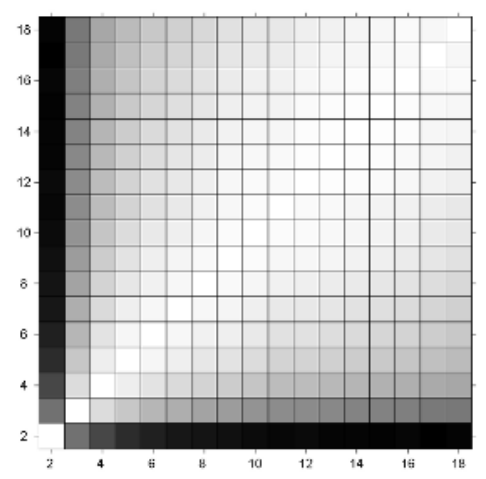

Fig. 5. Illustration of the correlation existing between the Cone-Curvatures of the wave fronts of orders 2 to 18 in the database

If we denote the WCC for each $N^{\prime} \in T^{\prime}$ as $c^{w}$, the linear combination will be:

$$
c^{w}=\sum_{j=1}^{q} v^{j} c^{j}
$$

where $v^{j}$ are the coordinates of the eigen vector associated with the eigen value of greater value of the covariance matrix for the $q$ initial variables.

This eigen vector was determined empirically by evaluating the principal components on the Cone-Curvatures of all the mesh nodes. As regards the orders considered, we studied three possibilities:

1. Wave fronts from $q=2$ to $q=18$.

2. Wave fronts from $q=4$ to $q=18$.

3. Wave fronts from $q=4$ to $q=9$.

Fig. 6 represents, for the object that we are analyzing, the Weighted ConeCurvatures in the three cases, plotted over the object mesh and using a color code to express the range from negative to positive values. We can see that the ConeCurvatures for the first and second cases are very similar.
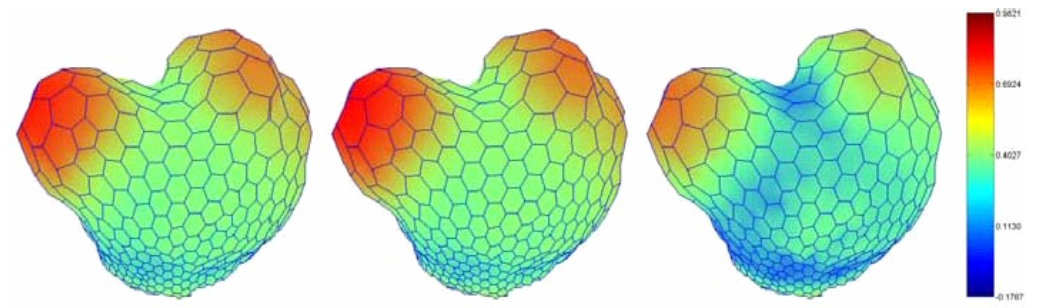

Fig. 6. Representation in color of the Weighted Cone-Curvatures of each mesh node for cases (1), (2) and (3) (left to right). A bar is shown where the colors can be seen for the maximum, mean and minimum Weighted Cone-Curvatures of the object. 


\subsection{Application of WCC to Partial Views Recognition}

In order to apply the WCC's to the recognition of partial views several factors must be taken into acount due to the nature of the handled meshes:

1. The number of complete wave fronts in a partial view is variable.

2. The surface represented by a set number of wave fronts can vary between a partial model and its complete model.

3. The mean length of the internode distance is different for the partial model and the complete model in the same object.

These questions imply that the CC's cannot be used as they are since the partial view and total view wave fronts cannot be compared because of the differences existing between the partial and total meshes. Therefore, we will define a measurement of error based on the WCC's:

Definition 1. Let $N^{\prime} \in T^{\prime}$ be the node of the partial mesh $T^{\prime}$ which is the nearest to the axis of vision. The error or distance of comparison of weighted cone-curvatures for each $N \in T_{i}$, where $T_{i}$ is the $i$-th total model of the object database is defined as:

$$
e_{j}(N)=\left|c^{w^{\prime}}\left(N^{\prime}\right)-c^{w}\left(N_{j}\right)\right|
$$

where the subscript $j$ extends from 1 to the maximum number of nodes existing in $T_{i}$ and $\mid \mathrm{I}$ represents the absolute value.

The fact of conditioning the reduction of nodes around $T_{i}$ to just one measurement of error can cause significant errors in this reduction. Therefore, in order to reinforce the reduction, for each $N \in T_{i}$ two errors will be measured. The first will consider the WCC's to the furthest fronts generated from $N$. We will call this error deep error and give it the symbol $e_{j}^{p}(N)$. The second will consider the nearest fronts generated from $N$. This time we will call the error superficial error and give it the symbol $e_{j}^{s}(N)$.

In both instances a set of errors equal to the number of nodes existing in $T_{i}$ is obtained, and from these errors the nodes $\mathbf{N}_{i}^{c c}$ of the mesh that will be passed to the next stage of the algorithm will be determined. If we call $\mathbf{N}^{p}$ to the set of nodes of $T_{i}$ with less $e_{i}^{p}$ values, and $\mathbf{N}^{s}$ to the set of nodes of $T_{i}$ with less $e_{i}^{s}$ values, $\mathbf{N}_{i}^{c c}$ will be:

$$
\mathbf{N}_{i}^{c c}=\mathbf{N}^{p} \cup \mathbf{N}^{s}
$$

\section{Principal Components and ICP Stages}

In this section the last two stages of the recognition algorithm will be commented on for matching the partial views on complete models. These are the principal components and ICP stages.

In the principal components stage the method proposed is based on calculating the principal components on the range data that we employed to obtain the model $T^{\prime}$ used in the previous stage. If we call these data $\boldsymbol{X}$, the principal components are defined as the eigen values and eigen vectors $\left\{\left(\lambda_{i}, \vec{e}_{i}\right) \quad i=1, \cdots, m\right\}$ of the covariance matrix. 
The eigen values are invariant to rotations and displacements and the eigen vectors to displacements. The eigen vectors conform a reference system linked to the data. This means that the eigen values can be used to evaluate what part of the range data of the complete model correspond to the scene, and the eigen vectors to calculate a first approximation to the transformation of the partial data to be matched on the total data. This approximation will only reflect the rotation sub matrix of the total transformation, since the origins of the two frames will coincide.

To apply this technique it is necessary to evaluate, before the recognition process, all the possibly existing partial views on the range data of the complete object. For this, the space of the possible points of view existing around the object was discretized, and a method was developed for generating virtual partial views (VPV's) based on the z-buffer algorithm. From each of these VPV their principal components will be calculated and used in the matching stage as explained earlier.

Comparison of the eigen values gives information about the possible areas where the partial view can be matched, but this information is global and, as we have said, only gives information about the rotation.

Thus it will be necessary to do a final calculation stage to refine the matching and to calculate the definitive transformation. For this we will use the ICP algorithm on a number of possible candidates marked in the eigen value comparison stage. The ICP must start from an approximation to the initial transformation, which in our case corresponds to the transformation given in the matching of the eigen vectors. The ending error in the ICP algorithm will measure the exactness of the definitive transformation and the correctness of the area where the view will be matched.

The comparison of the eigen values of the partial view and the virtual partial views is done by measuring an index of error given in the following expression:

$$
e_{i}^{c p}(N)=\left\|\Lambda_{i}^{v}(N)-\Lambda^{r}\right\|
$$

where $N \in \mathrm{N}_{i}^{c c}$ is the node from where we generated the $\operatorname{VPV}, \Lambda_{i}^{v}(N)$ is the vector formed by the eigen values of the VPV generated from the node $N$ of the i-th object of the object database $(i=1, \ldots, K), \Lambda^{r}=\left\{\lambda_{1}^{r}, \lambda_{2}^{r}, \lambda_{3}^{r}\right\}$ is the vector formed by the eigen values of the real partial view and $\|\cdot\|$ is the Euclidean distance.

After the error has been calculated for all the $\mathbf{N}_{i}^{c c}$ nodes and all the objects in the object database, we obtain a list of these errors, $e_{i}^{c p}(i=1, \ldots, K)$, ordered from least to great. If we compare the first error (least error for a set object) in all the lists, an ordering of the different objects in the object database will be obtained. Thus in the last stage we can apply the ICP algorithm on a subset of the object database, just $\mathbf{B}^{c p}$, and for each of the objects using the transformations associated with the subset of nodes that have produced these errors.

For the resolution of the ICP it is necessary to determine an approximation to the transformation matrix between the partial view and the object in the object database $\mathrm{R}_{1}$. This is calculated bearing in mind that the eigen vectors are orthonormal, and therefore:

$$
\mathbf{R}_{1}=\mathbf{E}^{r}\left(\mathbf{E}_{i}^{v}(N)\right)^{-1}=\mathbf{E}^{r}\left(\mathbf{E}_{i}^{v}(N)\right)^{T}
$$


where $\mathrm{E}_{i}^{v}(N)$ are the eigen vectors of the VPV generated from $N \in \mathrm{N}^{c p}$ of the i-th model of the object database $\mathrm{B}^{c p}$, and $\mathrm{E}^{r}$ are the eigen vectors of the partial view.

\section{Experimental Results}

The method proposed in this work was tested on a set of 20 objects. Range data of these objects have been acquired by means of a GRF-2 range finder sensor which provides an average resolution of approximately $1 \mathrm{~mm}$. Real size of the used objects goes from 5 to $10 \mathrm{~cm}$. height and there are both polyhedral shaped and free form objects (see Fig. 7). MWS Models have been built by deforming a tessellated sphere with 1280 nodes.

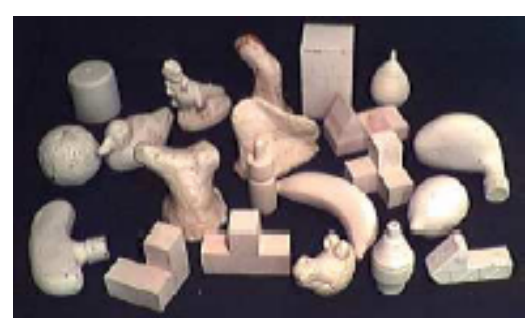

Fig. 7. Set of objects used to test the presented method

The recognition was done for three partial views per object, except in one of them where after the determination of its partial model it was seen that it did not have enough wave fronts to be able to compare the weighted cone-curvatures. This means that recognition was done on a total of 59 partial models. The success rate has been the $90 \%$, what demonstrates the validity of the method. The average computation time invested by the whole process was 90 seconds, programmed over a Pentium 4 at $2.4 \mathrm{GHz}$. computer under Matlab environment. A more detailed analysis of these results are next.

As it has been explained, in the first stage the weighted cone-curvatures of the partial model were compared for a node with a maximum number of wave fronts. From this comparison $\mathbf{N}_{i}^{c c}$ was determined (equation (3)). In the considered experiments, the maximum value of the number of nodes that form the sets $\mathbf{N}^{p}$ (deep search) and $\mathbf{N}^{s}$ (superficial search) was 32 each. Since the mesh used to obtain the complete model $T_{i}$ was 1280 , the minimum reduction of the space search in this stage was $95 \%$. The reduction can be even bigger as long as there are nodes coinciding in $\mathbf{N}^{p}$ and $\mathbf{N}^{s}$. This step was carried out for all the objects of the initial database $\mathbf{B}$ and took an average of 7.95 seconds.

Concerning the second stage, it started from these nodes and the eigen values were compared, which allowed us to achieve the first reduction of the database ( $\mathbf{B}^{c p}$ database). Reduction of the nodes obtained in the previous stage is also accomplished ( $\mathbf{N}_{i}^{c p}$ set). It was determined experimentally that $\mathbf{B}^{c p}$ consists of approximately $35 \%$ of the objects of $\mathbf{B}$ and $\mathbf{N}_{i}^{c p}$ of approximately $8 \%$ of the nodes of $\mathbf{N}^{c c}$ per object, which represent very satisfactory reduction rates of the stage. This process spent around $1 \mathrm{sec}$. 
Finally, the ICP algorithm was applied on seven objects (the mentioned 35\% remaining in the $\mathbf{B}^{c p}$ database) and three nodes (corresponding to the mentioned 8\%) for each $\mathbf{N}_{i}^{c p}$ object. As can be deduced, practically the most part of the time consumed for the algorithm was in this stage.

Fig. 8 shows some examples of recognition concerning to different shaped objects of the database. For each object, left side of this figure contains the range data corresponding to the partial view to recognize. Right side plots the range data of the partial view and the complete range data of the recognized object matched together in the position obtained after application of the algorithm.
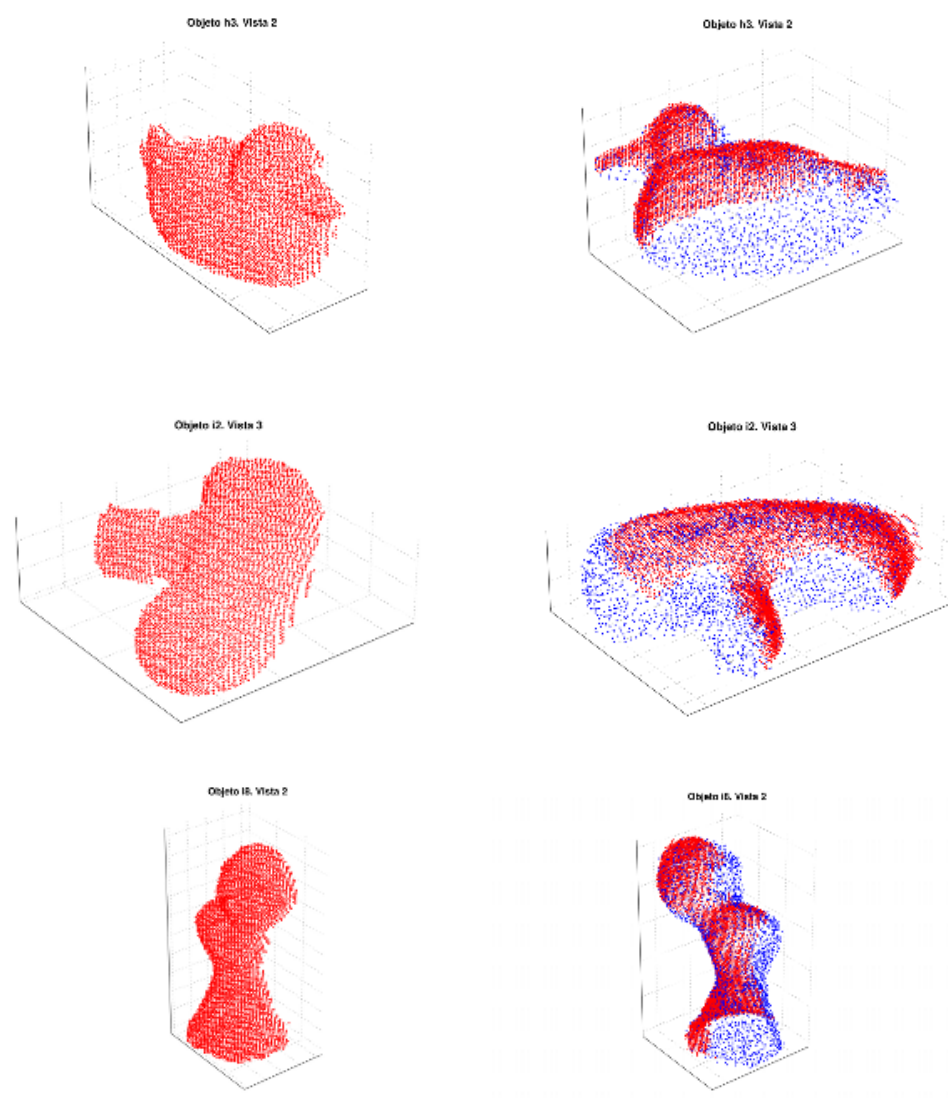

Fig. 8. Recognition results on free-form objects. On the left in each row the partial view to be recognized is shown and on the right the result obtained after applying the algorithm (both partial view and complete object range data).

\section{Conclusions}

This work has described a method for the recognition of free-form objects from their partial views. The method is divided into three stages: Weighted Cone-Curvatures 
stage, principal components stage, and ICP stage. Due to its novelty the first one has been described in more detail. The new feature WCC has been defined and analyzed. It exhibits the right properties for applying in recognition and positioning tasks. WCC's are calculated on the spherical models of the objects. These characteristics allow to achieve important reductions in the number of nodes that define the possible axes of vision from which the partial view has been acquired. The validity of the full method was proven with the recognition of 59 partial views in an object database of 20 objects. The success rate was $90 \%$.

We are currently working with a single view in real complex scenes where a hard and difficult task that frequently implies previous processes or steps must be solved before accomplishing recognition. In this case an effective $3 \mathrm{D}$ segmentation on the scene is essential to apply the recognition method presented in this paper.

\section{Acknowledgements}

This research has been carried out under contract with the Spanish CICYT through the DPI2002-03999-C02 project..

\section{References}

1. Adán, A., Adán, M.: A flexible similarity measure for 3D shapes recognition. IEEE Transactions on Pattern Analysis and Machine Intelligence, 26(11):1507-1520, November 2004.

2. Adán, A., Cerrada, C., Feliu, V.: Modeling wave set: Definition and application of a new topological organization of $3 \mathrm{D}$ object modeling. Computer Vision and Image Understanding, 79(2):281-307, August 2000.

3. Adán, A., Cerrada, C., Feliu, V.: Global shape invariants: a solution for 3D free-form object discrimination/identification problem. Pattern Recognition, 34(7):1331-1348, July 2001.

4. Campbell, R.J., Flynn, P. J.: Eigenshapes for 3D object recognition in range data. In Proc. of the IEEE Conference on Computer Vision and Pattern Recognition, volume 2, pages 2505-2510, Fort Collins, Colorado, June 1999.

5. Hebert, M., Ikeuchi, K., Delingette, H.: A spherical representation for recognition of freeform surfaces. IEEE Transactions on Pattern Analysis and Machine Intelligence, 17(7):681-690, July 1995.

6. Johnson, A. E., Hebert, M.: Using spin images for efficient object recognition in cluttered 3d scenes. IEEE Transactions on Pattern Analysis and Machine Intelligence, 21(5):433449, May 1999.

7. Skocaj, D., Leonardis, A.: Robust recognition and pose determination of 3-D objects using range image in eigenspace approach. In Proc. of 3DIM'01, pages 171-178, 2001.

8. Stein, F., Medioni, G.: Structural indexing: Efficient 3-D object recognition. IEEE Transactions on Pattern Analysis and Machine Intelligence, 14(2):125-145, February 1992.

9. Yamany, S., Farag, A.: Surfacing signatures: An orientation independent free-form surface representation scheme for the purpose of objects registration and matching. IEEE Transactions on Pattern Analysis and Machine Intelligence, 24(8):1105-1120, August 2002. 